\title{
A Review of Multidimensional Scaling (MDS) and its Utility in Various Psychological Domains
}

\author{
Natalia Jaworska and Angelina Chupetlovska-Anastasova \\ University of Ottawa
}

\begin{abstract}
This paper aims to provide a non-technical overview of multidimensional scaling (MDS) so that a broader population of psychologists, in particular, will consider using this statistical procedure. A brief description regarding the type of data used in MDS, its acquisition and analyses via MDS is provided. Also included is a commentary on the unique challenges associated with assessing the output of MDS. Our second aim, by way of discussing representative studies, is to highlight and evaluate the utility of this method in various domains in psychology.
\end{abstract}

The primary utility of statistics is that they aid in reducing data into more manageable pieces of information from which inferences or conclusions can be drawn. Multidimensional scaling (MDS) is an exploratory data analysis technique that attains this aim by condensing large amounts of data into a relatively simple spatial map that relays important relationships in the most economical manner (Mugavin, 2008). MDS can model nonlinear relationships among variables, can handle nominal or ordinal data, and does not require multivariate normality. As such, MDS provides an alternative to methods such as factor analysis and smallest space analysis, for example, in extracting representative information in data exploration (Johnston, 1995; Steyvers et al., 2002).

MDS provides a visual representation of dissimilarities (or similarities) among objects, cases or, more broadly, observations. In other words, the technique attempts to find structure in data by rescaling a set of dissimilarities measurements into distances assigned to specific locations in a spatial configuration (Giguère, 2006; Tsogo et al., 2000). As such, points that are closer together on the spatial map

Correspondence concerning this article should be addressed to: Natalia Jaworska, University of Ottawa, Institute of Mental Health Research, 1145 Carling Ave., Rm. 3128, Tel. 613.722.6521 \#6757 or 6297, E-mail: njawo040@uottawa.ca represent similar objects while those that are further apart represent dissimilar ones. The underlying dimensions extracted from the spatial configuration of the data are thought to reflect the hidden structures, or important relationships, within it (Ding, 2006; Young \& Hamer, 1987).

The logic of MDS analysis is most effectively introduced with an intuitive and simple example. For instance, if a matrix of distances between ten North American cities was subjected to MDS, a spatial map relaying the relative locations of these cities would be obtained (Figure 1). Cities that are physically close (more similar), are represented by points that are closer together on the map; the opposite is true for cities that are further apart. The emergent dimensions reflect geographical direction: one dimension corresponds to north-south while the other reflects eastwest.

In using MDS, the overall goal is to identify dimensions affecting perception or behavior, for instance, which may not have been readily evident in the data. This provides the analyst with a global overview of the relationships between variables. Such insight is highly valuable in psychological research dealing with qualitative data derived from scaling, sorting or ranking tasks as well as from questionnaires (Woosley et al., 2004).

The aim of this paper is to firstly provide a brief, nontechnical introduction of MDS, with a commentary on data collection, analyses and interpretation of the output. Detailed explanations of the mathematical bases of MDS, as 


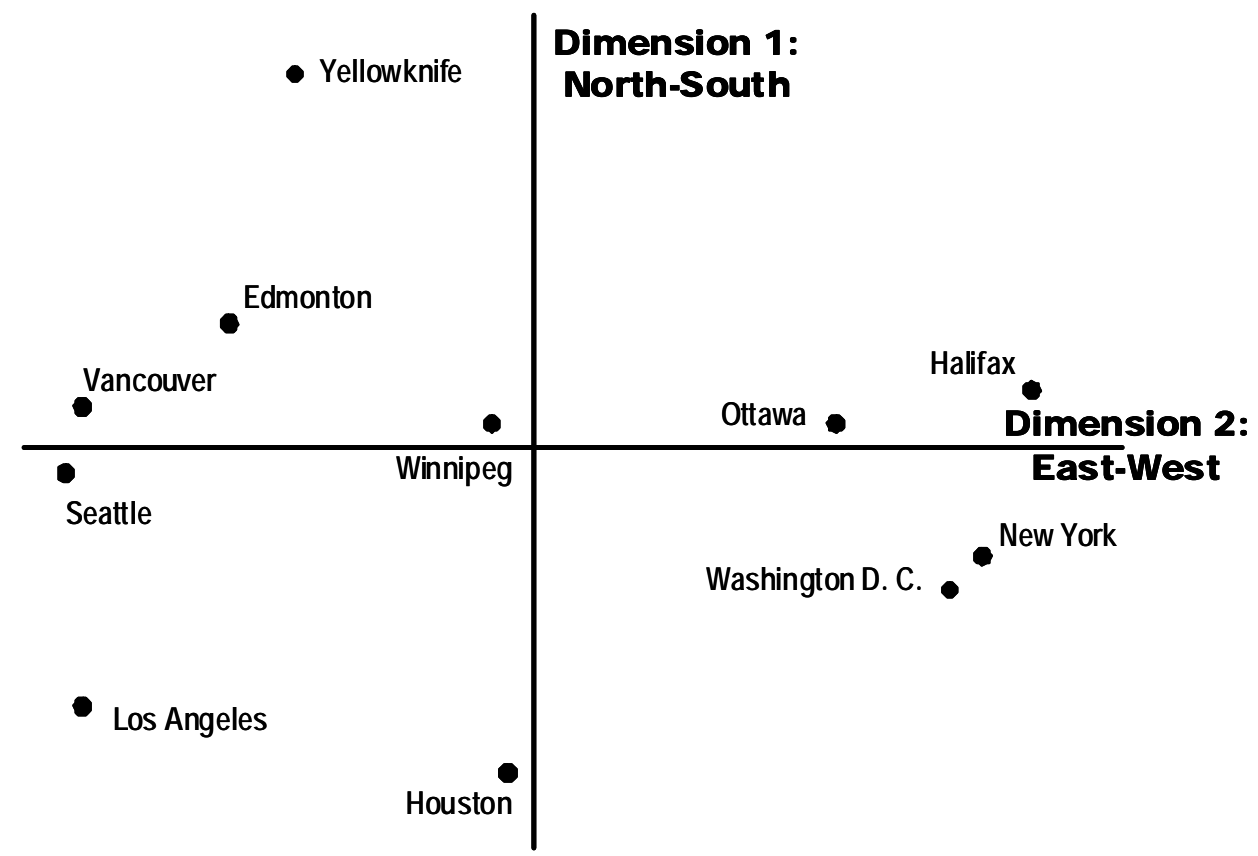

Figure 1. Example of a MDS solution applied to distances between pairs of cities.

well as assessments of statistical software packages used in carrying out MDS, are available elsewhere (e.g. Davidson \& Sireci, 2000; Giguère, 2006). Secondly, we will provide a synthesis of representative psychological work utilizing MDS analyses and assess the utility of this technique in psychology. MDS affords several advantages over other statistical methods commonly used in psychology: MDS is relatively simple to carry out and its visual output can be highly intuitive to interpret. The technique may also reveal findings not even considered during the formulation of original hypotheses. This is especially advantageous when relationships between factors are non-linear or multidimensional, as is often the case in psychology. Although MDS may lack the precision of other statistical techniques, it organizes data in a useful manner from which "first-glance" conclusions may be drawn (Davison, 1983).

\section{The Process of MDS}

\section{Data Types and Data Collection}

Broadly, the data used in MDS can be divided into two categories: Direct and indirect. Direct, also known as raw, data is obtained with techniques such as subjective sorting, ranking or rating of items, item comparisons or by creating item hierarchies. Data obtained from using Likert-type scales for rating the dis/similarity between color cards, for instance, is an example of direct data. Indirect, also known as derived or aggregate, data is computed from empirical measurements by correlations, associations or contingencies (Davidson, 1983). Confusion data is an example of indirect data that is derived from perceptual mistakes. Acquisition of confusion data can be illustrated with a hypothetical experiment where letters are briefly presented before a subject and the task is to identify them. Letters that are frequently confused with each other are rated as highly similar, while those that are rarely confused are highly dissimilar.

The data used in MDS can be referred to by several names (dissimilarities, similarities, distances, or proximities), with the terms "dissimilarity" and "similarity" data being most common. The distinction between the two lies in the types of scales used when rating differences between items. In similarity scales larger numbers indicate greater similarity, while in dissimilarity scales the opposite is true. For technical reasons, most MDS algorithms, like the Alternating Least-Squares Scaling (ALSCAL) algorithm in the Statistical Package for the Social Sciences (SPSS), are more efficient with dissimilarity measures. As such, data collected with the intention of subsequent MDS analyses is generally dissimilarity data.

Various data collection techniques for MDS exist; we will briefly outline the most common data collection methods. Direct data for MDS can be obtained through comparisons of all items within a set (e.g. "using a Likert scale, how dissimilar is item i from j?"). In pair-wise comparisons, the participant is asked to assess whether the dissimilarity between objects $i$ and $j$ is greater than between $k$ and 1 , for instance. When $\mathrm{n}$ (number of objects/pairs) is high, direct comparisons of all the items/item-pairs may make the task inefficient and unfeasible. As such, item comparisons are sometimes deleted either randomly or cyclically (Tsogo et al., 2000), with little impact on the final scaling solution even 
if up to a third of comparisons are removed (Rosett \& Klein, 1995). A faster alternative to direct comparisons is the use of grouping or sorting tasks, where participants sort items into k groups consisting of similar items (Tsogo et al., 2000). One variant of the sorting task is the hierarchical sorting task where the participant is asked to form a pre-specified number of groups and subsequently merges groups considered to be most alike. The merging process continues until all the objects are again contained within one group (Rao \& Kaltz, 1971). The dissimilarity between two objects, i and $j$, is therefore defined by the number of distinct groups in which $i$ and $j$ were grouped into. This information is then used to construct a proximity matrix, a table consisting of the dissimilarity data that serves as the input for MDS. Instead of sorting, participants can conduct ranking tasks. These require the designation of one reference item while the remaining items are treated as comparison objects. $\mathrm{K}$ objects are then ranked from most to least similar relative to the reference. The process is repeated until all the objects in the set have served as the reference (Rao \& Kaltz, 1971). Tsogo and colleagues (2000) provide an excellent description regarding how data obtained from various sorting and ranking tasks is converted into input proximities matrices that can then be used for MDS.

Traditionally, the aforementioned forms of data collection for processing with MDS were used in research on perception or in marketing research aimed at assessing consumer preferences (Bonebright et al., 1996; McIntyre \& Ryans, 1977). However, these data collection techniques have been adapted to assess the dimensions involved in more complicated cognitive phenomena, such as pain perception (Bertino \& Lawless, 1993; Knotkova et al., 2004), evaluation of emotions (Bimler \& Kirkland, 2001) as well as personality profiling (Ding, 2006). The appropriateness of choosing one data collection technique over another for the purpose of processing it via MDS is somewhat subjective, dependent on what the researcher wishes to assess, as well as the stimuli used (Bonebright et al., 1996). Subkoviak and Roecks (1976) have evaluated various forms of data collection approaches on the accuracy of the resulting multidimensional configuration of the examined objectspairs. Incidentally, they found significant differences in the multidimensional representations derived through different data collection techniques. As such, assessing the appropriateness of a data collection technique prior to commencing a study is warranted (Coxon \& Jones, 1979; Rao \& Katz, 1971).

\section{Types of MDS}

MDS is a generic term encompassing several different types of MDS procedures. These types can be classified according as to whether the input data is qualitative or quantitative, yielding non-metric and metric MDS, respectively. Metric MDS uses quantitative measurements of object comparisons (interval or ratio data). However, much of the data acquired in psychology is ordinal. As such, nonmetric MDS, which requires only qualitative information about dissimilarities, is more common in this field. The number of proximity (or similarity, dissimilarity, etc.) matrices and the nature of the MDS model are used in the classification of MDS subtypes. Classical MDS (CMDS) consists of a single matrix of either metric or non-metric data (Kruskal, 1964; Shepard, 1962; Torgerson, 1958). Replicated MDS (RMDS) deals with several matrices of dissimilarity data simultaneously but yields a single scaling solution, or one map (Steyvers, 2002). RMDS is typically used when data is obtained from several participants or one who is tested repeatedly, which is particularly useful for testing the stability of extracted dimensions. In weighted MDS (WMDS) the result is derived from several matrices that are assumed to differ from each other in systematically nonlinear or nonmonotonic ways. Since WMDS enables the model to account for individual differences in cognitive processes or perceptions, for instance, it is commonly referred to as the individual differences scaling (INDSCAL) model (Carroll \& Chang, 1970). MDS models are further elaborated when the input data consists of square asymmetric matrices. In this case, the rows and columns comprising a proximity matrix are the same objects but the proximity from observation $i$ to $j$ is not necessarily the same as from $j$ to $i$. Additional variants of MDS models and associated algorithms exist that deal with asymmetric proximity matrices, as well as with rectangular matrices. Commonly, a rectangular matrix consists of non-metric data in which the rows are stimuli and the columns are attributes. Our aim is to point out the existence of various MDS models, rather than to elaborate on them. As such, in our brief discussion of MDS model algorithms, output, and interpretation, we will limit our overview to the most basic version of MDS, namely CMDS, since all MDS models are variants of CMDS (for a detailed explanation of the various MDS models refer to Arce \& Gärling, 1989).

\section{The MDS Model and Output}

In non-metric MDS, stimuli are randomly represented in space and proximities between points are computed and transformed into disparities (distances) that preserve the order of the raw data using specific algorithms. The Minkowski distance model enables distances to be represented in geometrical space (Steyvers, 2002), with most MDS algorithms employing Euclidean principles. Where distance $\left(d_{i j}\right)$ between points $i$ and $j$ is defined as (Equation 
1):

$$
d_{i j}=\sqrt{\sum_{a}\left(x_{i a}-x_{j a}\right)^{2}}
$$

$x_{i}$ and $x_{j}$ specify coordinates of points $i$ and $j$ on dimension $a$, respectively. For non-metric data a positive monotone transformation is applied to dissimilarity data for scaling into spatial distances while for metric MDS a linear transformation function is applied (see Giguère, 2006, for details). Subsequently, a stress function that measures the fit between input proximities and distances is defined. An iterative process that attempts to find successive approximations to the solution is run until the stress function has been minimized (Arce \& Garling, 1989; Davidson, 1983; Kruskal \& Wish, 1978).

When running MDS analysis with statistical software such as SPSS or Statistical Analysis Software (SAS), the number of dimensions to be extracted from the spatial map must be pre-specified. As such, the researcher should have hypotheses regarding the number of expected dimensions from the data if the work is exploratory in nature, and especially if the aim of MDS is primarily for explanatory purposes. However, subsequently increasing or decreasing dimensionality to minimize stress is possible on all major software packages. When assessing the spatial map, one should also look for the existence of clusters. Clusters are groups of items (points on the map) that are closer to one another than to other items. These may represent a domain or sub-domain in the data, which may need to be analyzed separately.

\section{Output Diagnostics}

Stress indicates the difference between the input proximities and the output distances in the n-dimensional map. Kruskal's stress function (1964) is the most commonly used measure in determining a model's goodness of fit and is defined by (Equation 2):

$$
\text { Stress }=S=\sqrt{\frac{\sum_{i j}\left(\delta_{i j}-d_{i j}\right)^{2}}{\sum_{i j} d_{i j}{ }^{2}}}
$$

where $\delta_{i j}$ is the value of the proximities between items $i$ and $j$, and $d_{i j}$ is the spatial distance between them. Stress function values lay between zero and one; the smaller the stress function, the better the model represents the input data. Although there is no strict rule regarding how much stress is tolerable, the rule of thumb is that a value $\leq 0.1$ is excellent and anything $\geq 0.15$ is not tolerable (Kruskal \& Wish, 1978). Non-zero stress indicates that some, or all, distances in the map are, to some extent, distortions of the input data. Distortions may be spread over all relationships or be concentrated on a handful of points. As the number of dimensions increases, stress decreases, or stays the same, because with increasing dimensionality there is a closer fit between the input data and the model. However, increasing dimensionality decreases readability and interpretation of the MDS map. As such, the model may become nearly as complicated as the original data (Steyvers, 2002).

Another diagnostic tool for assessing the appropriateness of the MDS model is the squared correlation index $\left(R^{2}\right)$, which indicates the proportion of variance of the input data accounted for by the MDS procedure ( $R^{2} \geq 0.60$ is considered an acceptable fit; Meyer et al., 2005). The weirdness index is used in WMDS/INDSCAL and indicates how unusual each subject's weights are relative to the weights of the typical subject analyzed. The weirdness index varies from zero to one, where a score of zero indicates that the subject's weights are proportional to the average subject's weights. As the subject's score becomes more extreme the index approaches one, suggesting that optimal spatial configuration or scaling solution fits that subject poorly. Lastly, Shepard diagrams are scatterplots of input proximities ( $\mathrm{X}$ axis) against output distances ( $\mathrm{Y}$ axis) for every pair of items. In a perfect-fit model there should be no vertical discrepancy between the proximities and map distances.

\section{Interpreting and Validating MDS Output}

The primary objective of the analyst should be to obtain the best fit with the smallest number of possible dimensions. That said, although squeezing the input data into a two dimensional space enables "readability" it may be a very poor, highly distorted representation of the data, which is one caution that should be kept in mind when conducting MDS. One means of assessing if more dimensions are needed to better fit the data is by examining scree plots, which plot stress function value against dimension number. Ideally, there should be an obvious "elbow" within the scree plot indicating that increasing dimensions (right of the elbow) do not affect stress in any substantial way. As illustrated in Figure 2 (scree plot of theoretical data), after three dimensions there is no major reduction in stress. As such, the data can probably be represented effectively with three dimensions. In reality, the existence of a distinctive elbow is rare because stress typically declines smoothly with increased dimensionality, hence the utility of scree plots in identifying the most appropriate number of dimensions can be limited. High stress may also result from errors in measurement and sometimes the original data may need to be re-examined.

Although interpreting resultant dimensions is the task of the analyst, it is possible, albeit indirectly, to verify this 
interpretation. Resultant dimensions that emerge from MDS can be incorporated into regression analysis to assess their relationship with other variables (Green et al., 1989). Additionally, the stability of the MDS solution can be verified with a split-sample or multi-sample comparison, where the original sample is divided or a new sample collected, respectively. Generally, split-sample verification is favored as it is the more time- and cost-effective alternative. Furthermore, it can provide insight into whether a MDS solution is spurious or stable across similar samples.

\section{Trends in MDS Use in Psychology}

Using the search engines PsycINFO and PubMed, we synthesized journal articles in order to trace some of the trends in MDS use within the field of psychology. We focused our evaluation on articles of a psychological nature, excluding articles from the domains of medicine, genetics or neuroscience, as well as articles dealing directly with the theoretical aspects of MDS. Admittedly, this approach does not provide a complete, comprehensive review of MDS utility in psychology but rather enables the assessment of noticeable trends of MDS use in the field. These trends will be delineated by way of discussing representative studies.

\section{MDS Use in Test Construction and Validation}

MDS has been used in guiding test construction, evaluating test validity, as well as in modeling test responses. Item analysis is one of the primary procedures involved in constructing tests used in psychology, or in any field for that matter. According to Napier (1972), traditionally, item analysis has been unidimensional because items were selected on the basis of their covariation with the summary score. In order to avoid an early commitment to particular variables or underlying constructs, Napier suggested a non-metric, multidimensional analysis of items with MDS. He argued that MDS presupposes less stringent assumptions regarding the distributional and metric properties of the data. Addressing these points, Roth and Roychoudhury (1991) performed an item analysis on an anxiety questionnaire using MDS. The loading of items on the main factors in the questionnaire was revealed through analysis of item clusters in the MDS map, which was further confirmed with hierarchical cluster analysis. The analyses lead to the revision of the questionnaire with final reliability values of $\alpha=0.88-0.91$. As such, the authors advocated for the use of MDS in item analysis, which should produce more reliable testing tools.

MDS, much like factor analyses, can be applied in modeling test or item responses. Davidson and Skay (1991) consider factor analysis and MDS to be similar because both methods represent a continuous coordinate space from which structures called factors, in the one case, and dimensions, in the other, are extracted. The correlations and covariances produced by factor analysis can be seen as indices of proximity, and can thus be analyzed with MDS. Upon comparing MDS with factor analysis, Davidson and Skay (1991) concluded that conventional factor analysis of correlations or covariances provides a spatial representation of variability between individuals, while MDS provides a spatial representation of variability between tasks. Factor analysis, which is based on correlations among variables, accepts that scores vary along latent constructs, that observed responses are a linear function of a person's scores on those constructs, and that the sensitivity of tests to these constructs varies. MDS, however, assumes that most tests/tasks vary on several features, that individuals vary along each dimension according to their ideal point (one that represents the most preferred combination of perceived

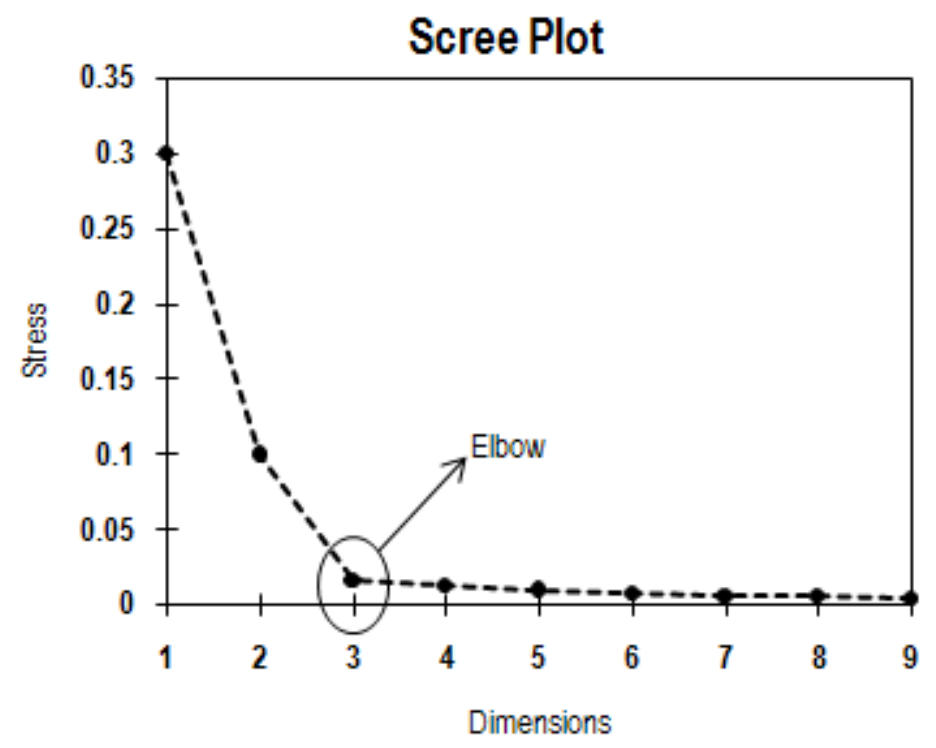

Figure 2. An example of a scree plot. 
attributes), and that the observed response of a person on a particular task is related to the difference between the features of the task and the subject's ideal point. As such, Davidson and Skay suggest using results from factor analysis within MDS for modeling test or item responses.

\section{Personality Profile Construction and MDS}

In constructing personality profiles with MDS the data obtained through personality assessment instruments is restructured and latent variables are derived. MDS represents them as dimensions, which are interpreted as profiles. According to Ding (2006) MDS enables representation of typical (normative) profiles within the population and simultaneously demonstrates how individuals differ with respect to these profiles. Individual variability along and across profiles is used to create an index of an individual's profile match. This index represents the extent to which an individual is represented by the normative profile. This approach to profile analysis is exploratory and is most suited to situations where normative profiles are derived from data rather than specified by a particular theory.

Kim and colleagues (2004) compared MDS with cluster analysis and modal profile analysis as methods used in profile analysis. Cluster analysis classifies objects into meaningful clusters or groups. The mean of each subtest score for all participants within the cluster describes the profile characteristics of that cluster. Modal profile analysis relies on standardized scores to yield clusters that vary in terms of profile shape. It identifies the most frequently occurring profile patterns in the data and compiles them to create normative profiles (Pritchard et al., 2000). One limitation of cluster analysis in comparison to MDS is that the clusters describe individual differences in overall profile level, rather than individual differences in profile patterns (Kim et al., 2004). Unlike MDS, modal profile analysis does not provide information regarding the level (average of all subtest scores) of profiles. An additional limitation of both cluster and modal profile analyses is that they can be difficult to apply to large sample sizes (Davidson \& Kuang, 2000). Hence, in addition to generating profile patterns, profile analysis with MDS provides information on profile level and efficiently analyzes samples of any size.

\section{Utility of MDS in Counseling Psychology}

Information acquired from the field of counseling psychology, from interviews, therapeutic sessions, projective techniques, etc., is multifaceted and often has no distinct structure. Additionally, this information is derived from a dynamic encounter between the counselor and client and thus lacks easily identifiable variables. Surface level analysis cannot account for all the underlying details of the data acquired within this field. Therefore, data analysis within counseling psychology benefits from methods like MDS. Fitzgerald and Hubert (1987) suggested analyzing individual differences by using multiple proximity matrices. These matrices may be obtained from identifiable subgroups or from individual subjects. Multiple measures may be generated if each subject's data is treated as a separate proximity matrix. Another approach is to group subjects on the basis of some salient variable, be it demographic or psychological, and construct proximity measures for each group. Additionally, separate proximity measures could be constructed for data collected at different times or in different settings. Thus, MDS can be utilized not only for representing interrelations between objects and determining underlying data dimensions, but also for providing a representation of individual or group differences. The practical value of a flexible approach in constructing various types of proximity matrices from the same data is that it offers maximum use of data and provides a greater wealth of information.

The psychology of individual differences, which focuses on a description of differences in psychological attributes among individuals as well as the antecedents and consequences of such differences, has had a large influence in counseling psychology (Dawis, 1992). Organizing individual difference variables most important within counseling psychology within some integrative framework can facilitate integration of the two fields. The work of Armstrong and colleagues (2008) addressed this need by developing the Atlas of Individual Differences. The purpose of the Atlas is to map the interrelations among measures of individual differences to facilitate career counseling (i.e. matching individuals with the best possible career path). Armstrong and colleagues utilized John Holland's theory of vocational choice in constructing the Atlas.

Holland's theory proposes that people choose jobs where they can be around people who are similar to themselves. It divides people into six personality types: realistic, investigative, artistic, social, enterprising, and conventional, which, if presented graphically, forms a two-dimensional hexagonal structure (Holland, 1997).

Armstrong and colleagues developed a threedimensional interest-based structure, which includes 31 environmental measures, to compare with Holland's hexagonal model. To integrate individual differences and environmental measures within Holland's model, Armstrong et al. used the linear multiple regression-based technique of property vector fitting (Kruskal \& Wish, 1978). This technique allows placement of external variables into a multidimensional space (i.e. Holland's model). Each fitted 
property vector summarizes the average relationship that exists between a specific cluster of external characteristics and Holland's model. The strength of a relationship is measured by the canonical correlation between the model's coordinates and the conditional probability scores of the variables in the cluster. A movement of the observed object towards the arrowhead of the vector is interpreted as reflecting an increasing tendency. Armstrong and colleagues felt that the results of their work demonstrate the appropriateness of MDS for creating a holistic model incorporating individual characteristics and environmental demands. The individual differences mapped in their work appear to be the product of a complex developmental process, whereby individuals find ways to function in their environment. Statistical methods, like MDS, that enable a representation of such complex relationships in a relatively intuitive manner may increase the potential effectiveness of integrating individual differences measurements in such applied settings as counseling psychology.

\section{Perception and MDS}

MDS is extensively used in studies dealing with perception. As such, we will limit our discussion to several interesting examples depicting the utility of MDS in this domain. With respect to olfactory perception, Lawless (1989) performed a study that aimed to assess odor classification by asking participants to sort odors, including ambiguous (also termed "boundary") ones. One group of participants sorted odors freely, while the other was restricted to sorting the odors into two categories. It was hypothesized that ambiguous odors would reveal more about the underlying dimensions of odor perception than unambiguous ones. The data from this procedure yielded a two-dimensional spatial map. Its coordinates were submitted to cluster analysis to confirm the results from MDS. For additional confirmation, a third experimental group completed the sorting task using objective criteria (e.g. intensity) for odor sorting. Their ratings were averaged and mean rating scale values for each odorant were regressed against their MDS map coordinates. The output of this regression was than used to generate direction cosines for vectors corresponding to the best-fitting projection of the rating scales into the MDS model. This procedure confirmed MDS pattern tendencies. Results from the free-sorting task revealed a circumflex structure in odor perception, with ambiguous odors in the center and distinct ones on the periphery. Restricted sorting clustered the odors into two groups with ambiguous scents represented within each group. As such, the production of a more detailed model depended on the flexibility of the sorting criteria granted to the participants, highlighting the necessity for careful consideration when choosing a data collection technique.

Bergmann Tiest and Kappers (2006) used MDS in the creation of haptic (tactile) perceptual space. Their research focused on tactile perception of materials encountered in an everyday context. Following free-sorting of the haptic stimuli, MDS analysis revealed a four-dimensional tactile perceptual space. This was validated with objective measurements of compressibility and roughness of the test materials. The objective measures were placed as external variables into the MDS model through property vector fitting. This procedure yielded moderate results, demonstrating that the objective physical characteristics of materials cannot fully explain the dimensionality of tactile space. Hence, a more complicated model within a curved non-Euclidean space may be necessary for better representation of tactile space, highlighting the limitation of Euclidian principle-based MDS. However, deviations from Euclidian principles and increased dimensionality may impede interpretation and intuitive understanding of the model.

\section{MDS and Perceptions of Emotion and Pain}

Since the experience of emotions is a highly subjective and qualitative phenomenon, the study of emotions is suited to analyses with non-linear statistical techniques like MDS. Izmailov and Sokolov (1999) performed an experiment that aimed to create a spatial representation of emotion. They presented subjects with schematic faces with systematically manipulated mouth and eyebrow angles that conveyed different emotions. Participants were asked to evaluate the difference between the faces, without naming the emotion, by using the method of semantic differentials. With this data, matrices of subjective differences for the faces were constructed. Subsequently, all subjective matrices were subtracted from each other and the emergent matrix of absolute values indicated the differences between emotions for all subjects. These absolute values were plotted in a hypothetical spherical space with dimensions consisting of emotional tone, intensity, and saturation. The experimenters then presented each emotional face along with labels of emotion to determine the correspondence between emotional space and its semantic label. This revealed a close association between the visual stimuli (emotional faces) and the semantic stimuli (emotion words), indicating that any change in the schematic emotional expression entailed a shift in its corresponding point in the spatial representation (Sokolov \& Boucsein, 2000).

Similarly to emotion, pain is a complex experience consisting of multiple attributes, such as somatosensory perception, tolerance, attitudes, etc., also suited for study with MDS. Currently, there is little consensus over the 
number and types of dimensions that may characterize pain perception (Boring, 1950). Clark and colleagues (1986) used the INDSCAL MDS model to explore the dimensions of thermal pain perception. Participants classified stimuli according to an intensity scale, with ratings ranging from no pain to significant unpleasant sensitivity (noxious). MDS analysis revealed a two dimensional "pain structure." The first dimension related to stimuli intensity, i.e. how weak or strong a stimulus feels. The second dimension was related to qualitative aspects of the stimuli, the pain-temperature attribute, which ranged from just detectable to painful, and from just detectably warm to hot. The authors argued that the bipolarity of the second dimension reflected randomness in the data. It was suggested that a third dimension may have been used by a few subjects with high weights. Subsequent work using MDS by the same group have elaborated on the two dimensional model, with the addition of more dimensions (Janal et al., 1991). According to Clark et al., $(1986 ; 1991)$ the salience of the dimensions of pain perception provides concept clarity, which should encourage further investigation between pain experience and individual difference parameters, such as anxiety, personality measures and mood.

\section{Concluding Remarks}

Multidimensional scaling (MDS) is an exploratory data analysis technique that can be used in testing the hypothesized existence of particular dimensions or structures within a data set. The use of MDS in data analyses offers several advantages. Namely, MDS is an extremely flexible technique, one that can model non-linear relationships and is not bound by the numerous assumptions associated with general linear models or even with factor analyses. That said, interpretation of MDS output can be challenging and is highly subjective. However, the dimensional outputs of MDS can be regressed with more objective variables, which can provide more confidence in the emergent scaling solution and its interpretation. Due to its flexibility and its relative freedom from strict theoretical boundaries, the use of MDS is evident in various psychological domains.

\section{References}

Arce, C., \& Garling, T. (1989). Multidimensional scaling. Anuario de Psicologia, 43, 63-80.

Armstrong, P. I., Day, S. X., McVay, J. P., \& Rounds, J. (2008). Holland's RIASEC Model as an Integrative Framework for Individual Differences. Journal of Counseling Psychology, 55(1), 1-18.

Bergmann Tiest, W. M., \& Kappers A. M. L. (2006). Analysis of haptic perception of materials by multidimensional scaling and physical measurements of roughness and compressibility. Acta Psychologica, 121(1), 1-20.

Bertino, M., \& Lawless, H. T. (1993). Understanding mouthfeel attributes: a multidimensional scaling approach. Journal of Sensory Studies, 8, 101-114.

Bimler, D. L., \& Kirkland, J. (2001). School Truants and Truancy Motivation Sorted out with Multidimensional Scaling. Journal of Adolescent Research, 16(1), 75-102.

Bimler, D. L, Kirkland, J., \& Jameson, K. A. (2004). Quantifying Variations in Personal Color Spaces: Are There Sex Differences in Color Vision? Color Research and Application, 29(2), 128-134.

Bonebright, T. L. (1996). An investigation of data collection methods for auditory stimuli: Paired comparisons versus a computer sorting task. Behavior Research Methods, Instruments, and Computers. 28, 275-278.

Boring, E. G. (1950). A history of experimental psychology. New York: Appleton-Century-Crofts.

Carroll, J. D., \& Chang, J. J. (1970). Analysis of individual differences in multidimensional scaling via an n-way generalization of "Eckart-Young" decomposition. Psychometrika, 35, 283-319.

Clark, W. C., Carroll, J. D., Yang, J. C., \& Janal, M. N. (1986). Multidimensional scaling reveals two dimensions of thermal pain. Journal of Experimental Psychology: Human Perception and Performance, 12(1), 103-107.

Coxon, A. P. M., \& Jones, C. L. (1979). Measurements and meanings: techniques and methods of studying occupational cognition. New York: St. Martin's Press.

Davison, M. L. (1983). Multidimensional Scaling. New York, New York: John Wiley \& Sons.

Davison, M. L., Gasser, M., \& Ding, S. (1996). Identifying major profile patterns in a population: An exploratory study of WAIS and GATB patterns. Psychological Assessment, 8, 26-31.

Davison, M. L., \& Kuang, H. (2000). Profile patterns: Research and professional interpretation. School Psychology Quarterly, 15(4), 457-464.

Davison, M. L., \& Sireci, S. G. (2000). Multidimensional scaling. In H. E. A. Tinsley \& S. D. Brown (Eds.), Handbook of applied multivariate statistics and mathematical modeling. San Diego, California: Academic Press.

Davidson, M. L., \& Skay, C. L. (1991). Multidimensional Scaling and Factor Models of Test and Item Responses. Psychological Bulletin, 110(3), 551-556.

Dawis, R.V. (1992). The individual differences tradition in counseling psychology. Journal of Counseling Psychology, 39(1), 7-19.

Ding, C. S. (2006). Multidimensional scaling modelling approach to latent profile analysis in psychological research. International Journal of Psychology, 41 (3), 226- 
238.

Fitzgerald, L. F., \& Hubert, L. J. (1987). Multidimensional Scaling: Some Possibilities for Counseling Psychology. Journal of Counseling Psychology, 34(4), 469-480.

Giguère, G. (2006). Collecting and analyzing data in multidimensional scaling experiments: A guide for psychologists using SPSS. Tutorial in Quantitative Methods for Psychology, 2(1), 27-38.

Green, P. E., Camone, F. J. Jr., \& Smith S. M. (1989). Multidimensional scaling: concepts and applications. Needham Heights, Massachusetts: Allyn and Bacon.

Heppner, P. P., Kivlighan, D. M., Burnett, J. W., Berry, T. R., Goedinghaus, M., Doxsee, D. J., Hendricks, M. F., et al. (1994). Dimensions That Characterize Supervisor Interventions Delivered in the Context of Live Supervision of Practicum Counselors. Journal of Counseling Psychology, 41(2), 227-235.

Holland, John. L. (1997). Making vocational choices: A theory of vocational personalities and work environments. Psychological Assessment Resources Inc.

Izmailov, C. A., \& Sokolov, E. N. (1991). Spherical model of color and brightness discrimination. Psychological Science, 2, 249-259.

Janal, M. N., Clark, W. C., \& Carroll, J. D. (1991). Multidimensional scaling of painful and innocuous electrocutaneous stimuli: Reliability and individual differences. Perception and Psychophysics, 50(2), 108-116.

Johnston, C. S. (1995). The Rokeach value survey: underlying structure and multidimensional scaling. The Journal of Psychology. 129(5), 583-597.

Kim, S. K., Frisby, C. L. \& Davison, M. L. (2004). Estimating Cognitive Profiles Using Profile Analysis via Multidimensional Scaling (PAMS). Multivariate Behavioral Research, 39(4), 595 - 624.

Knotkova, H., Crawford, C. W., Mokrejs, P., Padour, F., \& Kuhl, J. (2004). What do ratings on unidimensional pain and emotion scales really mean? A Multidimensional Affect and Pain Survey (MAPS) analysis of cancer patient responses. Journal of Pain and Symptom Management, 28(1), 19-27.

Kruskal, J. B. (1964). Nonmetric multidimensional scaling: A numerical method. Psychometrika, 29, 115-129.

Kruskal, J. B., \& Wish, M. (1978). Multidimensional scaling. Newbury Park, CA: Sage.

Lawless, H. T. (1989). Exploration of fragrance categories and ambiguous odors using multidimensional scaling and cluster analysis. Chemical Senses, 14(3), 349-360.

Loftus, G., Young, F. W., Null, C. H. \& Sarle, W. (1978). Interactive similarity ordering. Behavior Research Methods and Instrumentation, 10, 273-280.

Meyer, J. M., Heath, A. C., Eaves, L. J., \& Chakravarti, A.
(2005). Using multidimensional scaling on data from pairs of relatives to explore the dimensionality of categorical multifactorial traits. Genetic Epidemiology. 9(2), 87-107.

Moses, J. A. \& Pritchard, D. A. (1995). Modal profiles for the Wechsler Adult Intelligence Scale-Revised. Archives of Clinical Neuropsychology, 11, 61-68.

Mugavin, M. E. (2008). Multidimensional scaling: A brief overview. Nursing Research, 57(1), 64-68.

Napier, D. (1972). Nonmetric multidimensional techniques for summated ratings, In R, N. Shepard, A, K, Romney, \& S, B. Nerlove (Eds.), Multidimensional scaling: Vol. I, Theory. New York: Seminar.

Rao, V. R., \& Kaltz, R. (1971). Alternative multidimensional scaling methods for large stimulus sets. Journal of Marketing Research, VIII, 488-494.

Pritchard, D. A., Livingston, R. B., Reynolds, C. R., \& Moses, J. A. (2000). Modal profiles for the WISC-III. School Psychology Quarterly, 15, 400-418.

Rosett, T. R., \& Klein, B. P. (1995). Efficiency of a cyclic design and a multidimensional scaling sensory analysis technique in the study of salt taste. Journal of Sensory Studies, 10(1), 25-44.

Roth, W. M., \& Roychoudhury, A. (1991). Nonmetric multidimensional item analysis in the construction of an anxiety attitude survey. Educational and Psychological Measurement, 51(4), 931-942.

Sabers, D., Jones, P., \& Shirome, P. (1989). On methods for probing validity of intelligence tests: A commentary on the work of Zeidner and Feitelson. Journal of Psychoeducational Assessment, 7, 194-208.

Shepard, R. N. (1962). The analysis of proximities: Multidimensional scaling with an unknown distances function. Psychometrika, 27(2), 125-149.

Sokolov, E., \& Boucsein, W. (2000). A Psychophysiological Model of Emotion Space. Integrative Physiological and Behavioral Science, 35, 81-119.

Steyvers, M. (2002). Multidimensional scaling. In: Encyclopedia of cognitive science. Nature Publishing Group, London, UK.

Subkoviak, M., \& Roecks, A. L. (1976). A closer look at the accuracy of alternative data-collection methods for multidimensional scaling. Journal of Educational Measurement, 13(4), 309-17.

Torgerson, W. S. (1958). Theory and methods of scaling. New York: John Wiley and Sons.

Tsogo, L., Masson, M. H., \& Bardot, A. (2000). Multidimensional scaling methods for many-object sets: A review. Multivariate Behavioral Research, 35(3), 307-319.

Woosley, S. A., Hyman, R. E., \& Graunke, S. S. (2004). Q-sort and student affairs: A viable partnership? Journal of 
College and Student Development, 45(2), 231-242.

Young, F. W., \& Hamer, R. M. (1987). Multidimensional scaling: History, theory, and applications. Hillsdale, NJ: Lawrence Erlbaum Associates.
Zeidner, M., \& Feitelson, D. (1989). Probing the validity of intelligence tests for preschool children: A smallest space analysis. Journal of Psychoeducational Assessment, 7, 175-1.

Manucript received October $2^{\text {nd }}, 2008$

Manuscript accepted December 15 th 2008. 